\title{
Internet of Things Based Approach to Detect Obstacle in Fog to Avoid Accidents
}

\author{
${ }^{1}$ Rajesh Pandey, ${ }^{2}$ Avinav Pathak, ${ }^{3}$ Nidheesh Sharma, ${ }^{4}$ Rohit Vats \\ ${ }^{1}$ Assistant Professor, Shobhit Institute of Engineering \& Technology, Meerut, (Uttar Pradesh), \\ India \\ ${ }^{2}$ Assistant Professor, Shobhit Institute of Engineering \& Technology, Meerut, (Uttar Pradesh), \\ India \\ ${ }^{2}$ Assistant Professor, Dr. K. N. Modi Institute of Engineering \& Technology, Ghaziabad, (Uttar \\ Pradesh), India \\ ${ }^{2}$ Assistant Professor, Shobhit Institute of Engineering \& Technology, Meerut, (Uttar Pradesh), \\ India \\ ${ }^{1}$ rajesh@shobhituniversity.ac.in , 2avinav.pathak@shobhituniversity.ac.in , \\ 3nidheesh.sharma@knmiet.edu, ${ }^{4}$ rohit.vats@ shobhituniversity.ac.in
}

\begin{abstract}
Human life is of the utmost importance. Every year, many people lose their lives in road accidents. Reasons for an accident are many like over-speeding, poor traffic system, drink and drive, rough driving, etc. this paper aims to develop a model that can be used in vehicles to identify accidents due to Zero visibility in fog in winters and alarming for the same to avoid it. IoT is an emerging technology and can be intensively used for the intelligent transport system. This paper aims to develop an automated IoT enabled system to be used in car for the detection and avoidance of accidents due to zero visibility in fog. To build this system, the Ultrasonic sensor will be integrated with Raspberry Pi along with other supportive components (powerbank, Jumper wires, breadboard, etc.).
\end{abstract}

\section{Keywords}

IoT, Ultrasonic Sensors, Accident Avoidance, GPS, Raspberry Pi, Fog.

\section{Introduction}

Rapid growth in automobile industries and increasing population on earth leads to more and more traffic on the roads and this increases the accident cases involving human death. Every year, thousands of lives are lost in accidents that occur due to zero visibility in fog in the winter season. According to an article published by Times of India on Jan 5, 2019, the number of people dying in the year 2017 in fog-related road creases is 11,090. The objective of this paper is to save human lives from fog-related road accidents. The aim is to propose an IoT based designed system in cars to detect obstacles in fog so the human lives can be saved by avoiding accidents. To achieve car automation, we use Raspberry pi with a distance sensor and some supporting tools like Wi-Fi adapter, power backup, etc. Using a distance sensor, we'll be able to find out the actual distance of the obstacle in the real environment so that necessary actions or precautions can be taken to ensure the safety of lives. This IoT based system in cars can be used to monitor the data of the live scenario of the roads and alert for the emergency so that safety measure can be taken. 


\section{Review of Literature}

Over the ages, various alternatives were suggested to deal with the aforesaid problems. Here, a summary of the work done for the remedy of this problem is presented. Saraf et al. have given a method to calculate the distance between two vehicles and to know the traffic density in that particular area. His study aims to increase road safety by giving real-time alerts to the vehicle drivers about hurdles in their path and their nearby area. The main mechanism is the communication between the vehicle and the roadside unit [1]. Ismail et al. have established a technique to alert the driver of their respective vehicle while taking turns via a wireless communication system. The system uses a red light signal to alerts users of the other side lane to control the speed of the second vehicle to prevent collisions [2]. Leo et al. has suggested a mechanism to negotiate the problems of vehicles moving in hairpin bends in mountain areas. This mechanism gives priorities to the vehicles near the turnings, speed control, the indication of vehicle breakdown in the mountain areas, and accident alerts. GPS and GSM technologies are used for it [3]. Leo, Monisha, Sakthi, and Sunder et al. attempted to diminish the overhead from the drivers by placing an interface between the man and the machine [4]. Ueki, Mori, Nakamura, Horii, and Okada et al. in this paper defines a vehicular collision avoidance support system (VCASS), and evaluate performance by various tests with two real vehicles [5]. Kumar et al. has suggested an arrangement that talks about issues of unfavorable weather conditions such as heavy rain and massive snowfall. Rain and fog are forecasted by the humidity sensors. Once the unfavorable weather conditions are predicted, the next job is to avoid collisions [6]. MacHutchon et al. framed a system to identify fog and a cautioning framework based on the forward scatter principle was chosen. Following meteorological, traffic, and site overviews the fog threat index was applied and alarming messages will be shown inside the distinguished fog region[7]. Negru et al. have proposed a system to detect fog by using image processing. Estimation of distant visibility is based on the images taken from the camera of the moving vehicle in real-time [8]. Singhal et al. have designed a system that uses a GPS sensor system engaging Information and communication technology (ICT) for collision avoidance [9]. Mueller and Trick et al. has proposed a method that Examines the effects of driving experience and visibility on driving behavior [10]. de Bruyne et al. have designed a system with a $430 \mathrm{MHz}$ transceiver in cars coupled to the 4-way flasher control switch [11]. Ye, Adams, and Roy et al. have worked over the effect of an 802.11 based multi-hop Mac protocol that engenders an emergency warning message (EWM) down a detachment of cars on a roadway [12].

The aim is to design a system to establish wireless communication between several vehicle nodes to avoid accidents due to fog and on the blind courses. This system uses integrated RF and ultrasonic communication which results in providing alerts with minimal latency. The performance of the system may change depending on the operating conditions so it should be calibrated accordingly[13]. 


\section{Component Description}

\section{A. Raspberry Pi:}

Raspberry $\mathrm{Pi}$ is a fully functional credit card-sized computer that can be connected with a monitor. Various models of Raspberry Pi are available. We will use the Raspberry Pi B+ model. It has a $1.4 \mathrm{GHz}$ quad-core processor, dual-band wireless LAN, 4 USB 2.0 ports, full-size HDMI, and 1GB inbuilt RAM. The Raspberry Pi is a very cheap computer that runs Linux, but it also provides a set of GPIO (general purpose input/output) pins that allow you to control electronic components for physical computing and explore the Internet of Things (IoT).

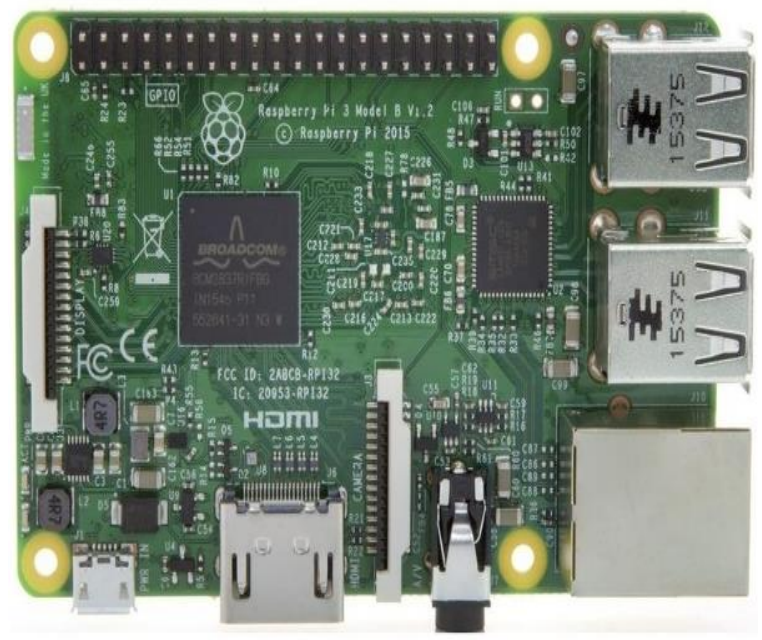

\section{B. Distance Sensor: Ultrasonic sensor (HC-SR04):}

An ultrasonic sensor, shown in Figure 2, is used to measure the distance of any obstacle from the car. It transmits a high-frequency sound wave and receives back its echo. It calculates the time taken between the transmission of signal and receiving of its echo to determine the distance to an object.

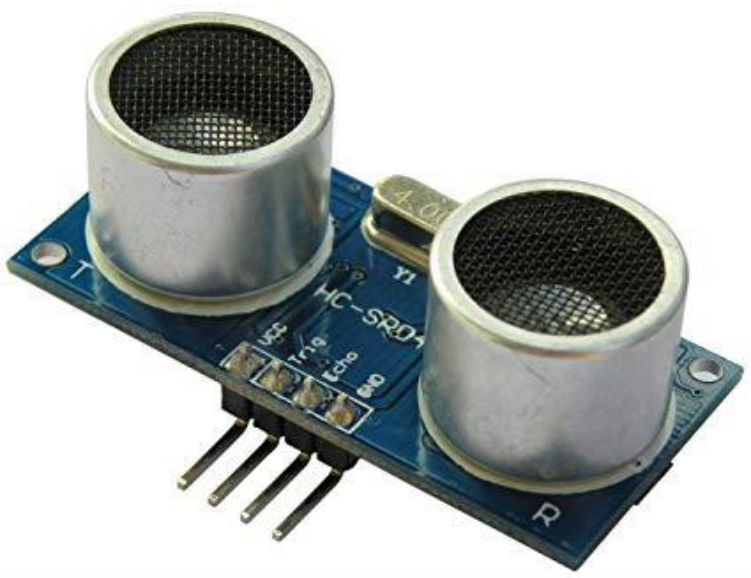

Figure 2. Ultrasonic Sensor: HC-SR04 


\section{GSM module:}

A GSM (Global System for Mobile Application) module is a chip or circuit which is used for the communication between a mobile device or a computing machine and a GSM system.

\section{GPS module:}

GPS (Global Positioning System)is a device that is used for receiving information from GPS satellites and then to locate the device's geographical position. Coordinates of the vehicle are delivered to the pre-registered phone number with the help ofthe GSM module.

\section{Proposed System}

In this proposed system, an IoT based system will be installed in the car. It will guide and navigate the drivers to drive their car securely and properly. This system will use the GPSfor tracking and locating the accident location and an onboard GPS module is used tocommunicate the coordinates via SMS.The distance of the obstacle in front and rear of the car is measured by an Ultrasonic sensor. The main processing unit of the system is the Raspberry pi board which handles all data accumulation, analysis of that data, and taking actions based on that data.

\section{A. Architecture:}

Ultrasonic sensors are placed at the front and rear of the car to detect the obstacle. The system consists of a GSM (Global System for Mobile communication) and GPS (Global Positioning System) for emergency assistance, in the event of any emergency positional data which is obtained from GPS module is communicated to a pre-registered phone number via SMS using GSM module. All the sensors and modules are connected in immediate withthe raspberry pi board to its GPIO (General Purpose Input Output) pins, and a centralized program monitors sensors and issues actions based on their status. The architecture of the proposed system is shown in Figure 3.

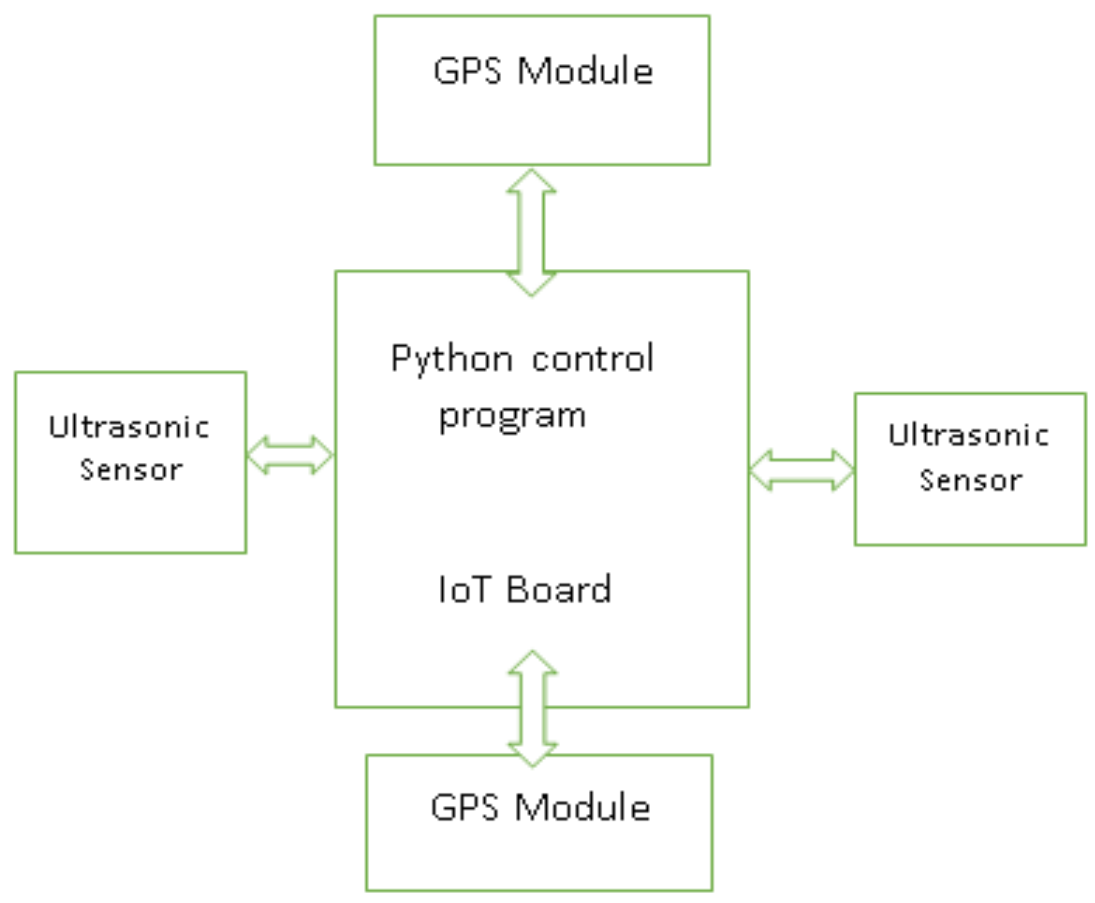

Figure 3. The architecture of the proposed model 


\section{B. User Interface:}

The system consists of a GUI interface programmed and developed with Python. There are buttons to activate Ultrasonic sensors which are integrated with the raspberry pi. Sensors continuously monitor the road by transmitting signals, and when a notification has to be displayed, it is displayed in the road status part of the main window. It has a set of buttons to activate and input data can be provided to logical sensors. When a button is pressed a pop-up window appears with a text box for data reception, and after the data is processed status of the particular sensor is displayed in a status window.

\section{Distance Calculation:}

Let us consider that $\mathrm{d}$ be the distance between the emitter and the receiver. $\mathrm{A}$ is the angle made at the obstacle by the incident and reflected signals. $\mathrm{D}$ is the exact distance of the obstacle from the car is given by the equation (1).

$$
\mathrm{D}=\mathrm{d} / 2 \tan (\alpha / 2)
$$

Figure 4 shows the block diagram of the system that detects obstacle and calculate the exact distance D between obstacle and the vehicle.

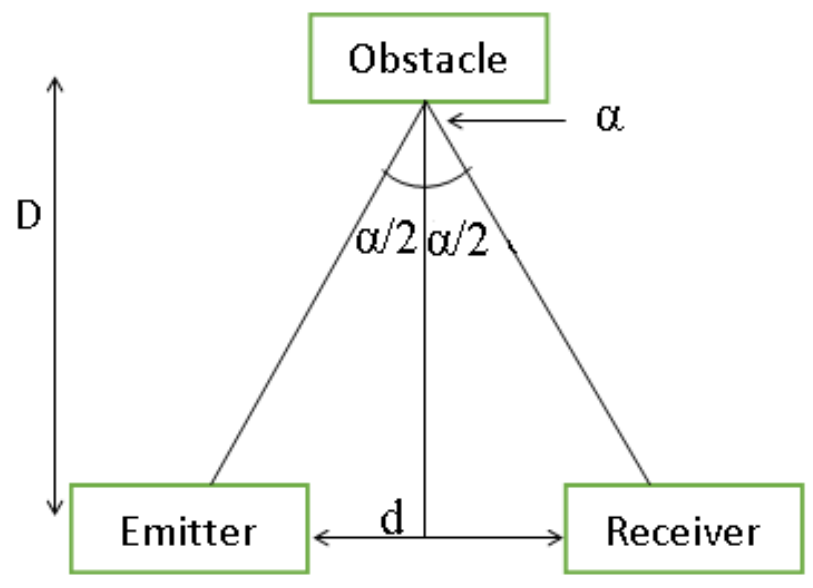

Figure 4. Block diagram of proposed system

D. Algorithm:

Obstacle Detection

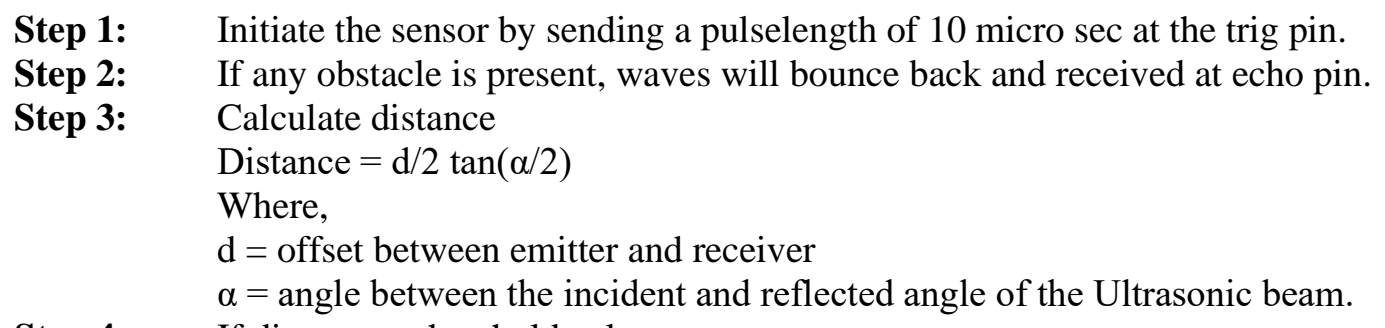

Step 4: $\quad$ If distance < threshold value 
Alert driver

I.

\section{CONCLUSION}

In recent years, a large number of road accidents occurred involving human death due to dense fog in winter where visibility is almost zero. So, more advanced technologies may be applied to implement a safety system. This system is one step in the direction of safety of lives while driving vehicles. The components used (Raspberry Pi, sensors, GPS, and GSM module) are cheap and reliable. Raspberry pi is used as a central controlling board and is interfaced with sensors for obstacle detection; board uses data from these sensors to avoid accidents or may ask for assistance in emergencies using a GSM module with coordinates from a GPS module.

\section{References}

[1]. Saraf, P.D., Chavan, N.A.: Pre-crash sensing and warning on curves: a review. Int. J. Latest Trends Eng. Technol. (IJLTET) 2(1) (2013)

[2]. Ismail, M., et. al.: Intersection cross-traffic warning system for vehicle collision avoidance. Int. J. Adv. Res. Electr. Electron. Instrum. Eng. 3, 13155-13160 (2014). https://doi.org/10. 15662/ijareeie.2014.0311031

[3]. Leo, J.J., et. al.: Vehicle movement control and accident avoidance in a hilly track. In: 2014 International Conference on Electronics and Communication Systems (ICECS), pp. 1-5. IEEE (2014)

[4]. Leo, J., Monisha, R., Sakthi, B.T.S., Sunder, A.C.: Vehicle movement control and accident avoidance in a hilly track. In: 2014 International Conference on Electronics and Communication Systems (ICECS) (2014). https://doi.org/10.1109/ecs.2014.6892757

[5]. Ueki, J., Mori, J., Nakamura, Y., Horii, Y., Okada, H.: Development of vehicular-collision avoidance support system by inter-vehicle communications - VCASS. In: 2004 IEEE 59 $9^{\text {th }}$ Vehicular Technology Conference VTC 2004-Spring (IEEE Cat. No. 04CH37514). https://doi.org/10.1109/vetecs.2004.1391463

[6]. Kumar, T.A.S., Mrudula, J.: Advanced accident avoidance system for automobiles. Int. J. Comput. Trends Technol. (IJCTT) 6(2), 79-83 (2013)

[7]. MacHutchon, K.R., Ryan, A.: Fog detection and warning, a novel approach to the sensor location. In: 1999 IEEE African. 5th Africon Conference in Africa (Cat. No. CH36342), vol. 1, pp. 43-50. IEEE (1999)

[8]. Negru, M., Nedevschi, S.: Image-based fog detection and visibility estimation for driving assistance systems. In: 2013 IEEE 9th International Conference on Intelligent Computer Communication and Processing (ICCP), pp. 163-168. IEEE (2013) Accident Avoidance System in Blind Curves and Fog 133

[9]. Singhal, V., Jain, S.S.: A novel forward vehicle collision avoidance methodology through the integration of information and communication technologies at intersections (2012)

[10]. Mueller, A., Trick, L.: Driving in fog: The effects of driving experience and visibility on speed compensation and hazard avoidance. Accid. Anal. Prev. 48, 472-479 (2012) 
[11]. deBruyne, P.: Fog collision-avoidance warning device. In: Proceedings IEEE 31st Annual 1997 International Carnahan Conference on Security Technology. https://doi.org/10.1109/ccst.1997.626264

[12]. Ye, F., Adams, M., Roy, S.: V2 V wireless communication protocol for rear-end collision avoidance on highways. ICC Workshops - 2008 IEEE International Conference on Communications Workshops (2008). https://doi.org/10.1109/iccw.2008.77

[13]. Singh A., Ratnakar R., Rajak A., Gurale N., Pacharaney U.S. (2020) Accident Avoidance System in Blind Curves and Fog Using Integration of Technologies. In: Karrupusamy P., Chen J., Shi Y. (eds) Sustainable Communication Networks and Application. ICSCN 2019. Lecture Notes on Data Engineering and Communications Technologies, vol 39. Springer, Cham. https://doi.org/10.1007/978-3-030-34515-0_13

[1] 